\title{
STRONG COMPLETE MINORS IN DIGRAPHS
}

\author{
MARIA AXENOVICH ${ }^{1}$, ANTÓNIO GIRÃO $^{2}$, RICHARD SNYDER $^{1}$, AND LEA WEBER $^{1}$
}

\begin{abstract}
Kostochka and Thomason independently showed that any graph with average degree $\Omega(r \sqrt{\log r})$ contains a $K_{r}$ minor. In particular, any graph with chromatic number $\Omega(r \sqrt{\log r})$ contains a $K_{r}$ minor, a partial result towards Hadwiger's famous conjecture. In this paper, we investigate analogues of these results in the directed setting. There are several ways to define a minor in a digraph. One natural way is as follows. A strong $\vec{K}_{r}$ minor is a digraph whose vertex set is partitioned into $r$ parts such that each part induces a strongly-connected subdigraph, and there is at least one edge in each direction between any two distinct parts. We investigate bounds on the dichromatic number and minimum out-degree of a digraph that force the existence of strong $\vec{K}_{r}$ minors as subdigraphs. In particular, we show that any tournament with dichromatic number at least $2 r$ contains a strong $\vec{K}_{r}$ minor, and any tournament with minimum out-degree $\Omega(r \sqrt{\log r})$ also contains a strong $\vec{K}_{r}$ minor. The latter result is tight up to the implied constant, and may be viewed as a strong-minor analogue to the classical result of Kostochka and Thomason. Lastly, we show that there is no function $f: \mathbb{N} \rightarrow \mathbb{N}$ such that any digraph with minimum out-degree at least $f(r)$ contains a strong $\vec{K}_{r}$ minor, but such a function exists when considering dichromatic number.
\end{abstract}

\section{INTRODUCTION}

The relationship between the chromatic number of a graph and the existence of certain types of substructures, like minors and topological minors, has a long history. As usual, we say that a graph is a $K_{r}$ minor if there is a partition of its vertex set into $r$ parts such that each part induces a connected subgraph, and there is at least one edge between any two distinct parts. One of the most famous examples of a problem of the aforementioned type is Hadwiger's conjecture: for every $r \geq 1$, any graph with chromatic number at least $r$ contains a $K_{r}$ minor.

There are a few partial results concerning this conjecture. It is known to be true for $r \leq 6$ (see $[4,8,21,24])$. For general $r$, Kostochka [15] and Thomason [22] independently showed that any graph with average degree $\Omega(r \sqrt{\log r})$ contains a $K_{r}$ minor. Of course, this implies that any graph with chromatic number $\Omega(r \sqrt{\log r})$ contains a $K_{r}$ minor. This was the best general result towards Hadwiger's conjecture for some time. Recently, however, Norin, Postle, and Song $[17,19]$ improved this result. Building off of that work, Postle [20] proved that any graph with chromatic number $\Omega\left(r(\log \log r)^{6}\right)$ contains a $K_{r}$ minor, and this is the best bound to date.

In this paper, we look at analogous problems in the directed setting. In particular, we investigate the existence of certain types of minors in digraphs under conditions such as large dichromatic number and large minimum out-degree. Before proceeding, let us introduce a bit of terminology. We remark that all digraphs we consider are simple and do not contain loops. A digraph $D$ is strongly-connected if for every ordered pair $(u, v)$ of vertices in $D$ there is a directed path in $D$ from $u$ to $v$. As usual, $D$ is weakly-connected if its underlying graph is connected. The complete directed graph on $r$ vertices, denoted by $\vec{K}_{r}$, is a directed graph in which every pair of vertices is connected by an edge in each direction.

\footnotetext{
2 School of Mathematics, University of Birmingham, Edgbaston, Birmingham, B15 2Tt, United KINGDOM.

${ }^{1}$ Department of Mathematics, Karlsruhe Institute of Technology, 76131 Karlsruhe, Germany. E-mail addresses: maria.aksenovich@kit.edu, giraoa@bham.ac.uk, richard.snyder@kit.edu, lea.weber@kit.edu.

Date: 13th October 2020.

2 The research leading to these results was also partially supported by the EPSRC, grant no. EP/N019504/1 (A. Girão).
} 
There are several ways one can define a minor in a digraph (e.g., the notion of a butterfly minor, originally introduced by Johnson, Robertson, Seymour, and Thomas; see section 5 of [13]). Here, we consider so-called strong minors. Given a digraph $H$, we say that $D$ is a strong $H$ minor if $V(D)$ admits a partition $\left\{X_{v}: v \in V(H)\right\}$ into non-empty sets (called branch sets) such that

- the digraph $D\left[X_{v}\right]$ induced by $X_{v}$ is strongly-connected for all $v \in H$, and

- $u v \in E(H)$ if and only if there is an edge in $D$ from $X_{u}$ to $X_{v}$.

A similar definition holds for weak $H$ minors: we merely insist in this case that each branch set induces a weakly-connected subdigraph. We say that a digraph $D$ contains a strong (weak) $H$ minor if it contains a strong (weak) $H$ minor as a subdigraph. Equivalently, $D$ contains a strong $H$ minor whenever $H$ can be obtained from a subdigraph of $D$ by repeatedly contracting a strongly-connected subdigraph to a vertex and removing loops and multiple edges. We remark that the notion of a strong minor has been investigated before by Kim and Seymour [14], where they showed that tournaments (more generally, semi-complete digraphs) are well-quasi-ordered under the strong minor relation.

Jagger ([11], [12]) investigated extensions of the classical result of Kostochka and Thomason to digraphs. In particular, he addressed the following question: How many edges must a digraph have in order to guarantee weak (or strong) complete minors as subdigraphs? In particular, he showed [11] that any digraph with average degree $\Omega(r \sqrt{\log r})$ contains a weak $\vec{K}_{r}$ minor. This is a weak-minor analogue of the aforementioned result of Kostochka and Thomason. A similar theorem, however, cannot hold for containing strong $\vec{K}_{r}$ minors: the transitive tournament on $n$ vertices has $\left(\begin{array}{l}n \\ 2\end{array}\right)$ edges, but does not even contain a strong $\vec{K}_{2}$ minor. Thus, in order for a digraph $D$ to contain a strong $\vec{K}_{r}$ minor, it must have more than $\left(\begin{array}{c}|D| \\ 2\end{array}\right)$ edges. Another way of saying this is the following: There is no function $f: \mathbb{N} \rightarrow \mathbb{N}$ such that any digraph with average degree at least $f(r)$ contains a strong $\vec{K}_{r}$ minor. This suggests that density is not the appropriate digraph parameter to force strong complete minors, but perhaps there are other parameters that do so.

A $k$-colouring of a digraph is a partition of its vertex set into $k$ acyclic sets. The minimum $k$ for which this is possible is the dichromatic number of $D$, which we shall denote by $\chi(D)$ (as we never consider the usual chromatic number in this paper, we hope this causes no confusion). This parameter was introduced by Neumann-Lara [16], and has garnered interest in recent years (e.g., see $[2,9,10]$ for some interesting results concerning this parameter).

While the transitive tournament discussed above is dense, it contains no large complete minors. On the other hand, there are two parameters for which the transitive tournament is essentially trivial: it only has minimum out-degree 0 and dichromatic number 1 . Therefore, one might hope that large dichromatic number/out-degree is sufficient to guarantee large complete minors in general digraphs. And, if this is not the case, perhaps it is true in the more restrictive class of tournaments. In general, which digraph parameters, if sufficiently large, force large strong complete minors? This is the kind of question we address in this paper, our focus being on large dichromatic number and large minimum out-degree. We remark that this type of problem has been addressed in the context of forcing subdivisions in digraphs, instead of minors; see Aboulker, Cohen, Havet, Lochet, Moura, and Thomassé [1], and the recent results of Gishboliner, Steiner, and Szabó [6,7].

1.1. Our results. We shall introduce the following terminology in order to state our results:

Definition 1.1. For a digraph $D$, let $\operatorname{sm}(D)$ denote the largest $r$ such that $D$ contains a strong $\vec{K}_{r}$ minor.

Our aim is to determine whether large dichromatic number or large out-degree is sufficient to force the existence of strong $\vec{K}_{r}$ minors in digraphs. We show that the large dichromatic number indeed guarantees the existence of strong $\vec{K}_{r}$ minors in digraphs. However, we show that the large minimum out degree is not sufficient to force strong minors in general digraphs, but it is sufficient in tournaments. Specifically, we extend the classical results of Kostochka and 
Thomason to strong minors: any tournament with minimum out-degree $\Omega(r \sqrt{\log r})$ contains a strong $\vec{K}_{r}$ minor.

We first consider tournaments. The following theorem asserts that dichromatic number linear in $r$ already forces strong $\vec{K}_{r}$ minors in tournaments.

Theorem 1.2. Let $r \geq 1$ be an integer and suppose $T$ is a tournament with $\chi(T) \geq 2 r$. Then $\operatorname{sm}(T) \geq r$. Moreover, for every $r \geq 2$ there exists a tournament $S_{r}$ such that $\chi\left(S_{r}\right)=r$ and $\operatorname{sm}\left(S_{r}\right) \leq r-1$.

The construction of $S_{r}$ is done in Section 4. We believe that this construction is closer to the truth, concerning the correct dependence on $\chi$ for finding strong complete minors.

As is customary, for a digraph $D$ we denote by $\delta^{+}(D)$ the minimum out-degree of $D$. Our next theorem investigates strong complete minors in tournaments with large minimum out-degree. It may be viewed as the appropriate analogue of the classical result of Kostochka and Thomason for strong complete minors.

Theorem 1.3. There exists a constant $C>0$ such that the following holds. If $r$ is a positive integer and $T$ is a tournament with $\delta^{+}(T) \geq C r \sqrt{\log r}$, then $\operatorname{sm}(T) \geq r$. Moreover, this is tight up to the constant $C$.

The tightness can be seen by considering a random tournament on $c r \sqrt{\log r}$ vertices, for some constant $c>0$, and applying a standard argument (e.g., see Bollobás, Catlin and Erdős [3]). In fact, this argument shows that the random tournament on $c r \sqrt{\log r}$ vertices with high probability does not even contain $r$ nonempty sets with an edge in each direction between each pair of sets. The analogous result to Theorem 1.3 for digraphs is false, however:

Theorem 1.4. There is no function $f: \mathbb{N} \rightarrow \mathbb{N}$ such that every digraph $D$ with $\delta^{+}(D) \geq f(r)$ satisfies $\operatorname{sm}(D) \geq r$.

In particular, we show that a construction due to Thomassen [23] has large minimum outdegree, but does not even contain strong $\vec{K}_{3}$ minors; we prove this in Section 4 (see Proposition 4.3). On the other hand, we can show that large dichromatic number is sufficient to force strong minors in general digraphs.

Theorem 1.5. Let $r \geq 1$ be an integer. If $D$ is a digraph with $\chi(D) \geq r 4^{r}$, then $\operatorname{sm}(D) \geq r$.

Note that according to the second statement of Theorem 1.2 there is a digraph $S_{r}$ such that $\chi\left(S_{r}\right)=r$ and $\operatorname{sm}\left(S_{r}\right) \leq r-1$. The digraph $S_{r}$ is a tournament. In addition, we show in Section 4 that for every $r \geq 2$ there exists a digraph $G_{r}$ with $\chi\left(G_{r}\right)=r$ and $\operatorname{sm}\left(G_{r}\right) \leq r-1$. In comparison with $S_{r}$, the digraph $G_{r}$ is quite sparse. We believe the exponential dependence on $r$ in the first part of this theorem is far from the truth.

1.2. Organization. The remainder of this paper is organized as follows. Section 2 is devoted to additional definitions and notation. In Section 4 we analyze constructions of a tournament and digraph that have dichromatic number $r$, but do not contain strong clique minors on $r$ vertices. We additionally prove Theorem 1.4 by analyzing Thomassen's construction. In Section 5 we prove Theorem 1.2 and Theorem 1.3, which concern finding strong minors in tournaments under the assumption of large dichromatic number and large minimum out-degree, respectively. In Section 6, we prove our results concerning digraphs. First, we show that the assumption of large minimum out-degree is not sufficient to guarantee strong clique minors. Second, we prove Theorem 1.5, which shows that large dichromatic number is sufficient. Finally, we conclude in Section 7 with some remarks and open problems.

\section{Notation And TERMinology}

Here we provide some additional notation and terminology that will be used throughout the paper. Any further notation shall be introduced as necessary.

Let $D$ be a digraph. We denote by $V(D)$ and $E(D)$ the vertex and edge set of $D$, respectively. For a vertex $v$ in $D$, we write $N_{D}^{+}(v)$ and $N_{D}^{-}(v)$ for the out-neighbourhood and in-neighbourhood 
of $v$ in $D$, respectively. We let $d_{D}^{+}(v)=\left|N_{D}^{+}(v)\right|$ and $d_{D}^{-}(v)=\left|N_{D}^{-}(v)\right|$ denote the out-degree and in-degree of $v$. We let $\delta^{+}(D)=\min _{v \in V(D)} d_{D}^{+}(v)$ denote the minimum out-degree of $D$. We shall always omit the subscript ' $D$ ' when the digraph is clear from context. Given a subset $X \subseteq V(D)$, we denote by $D[X]$ the subdigraph of $D$ induced by $X$. We say that a subset of vertices $X \subseteq V(D)$ is acyclic if the subdigraph $D[X]$ induced by $X$ contains no directed cycle. Alternatively, we may say that $X$ (or the induced subdigraph $D[X]$ ) is transitive.

For disjoint sets $X, Y \subseteq V(D)$, we write $X \rightarrow_{D} Y$ provided every edge of $D$ with an endpoint in $X$ and an endpoint in $Y$ is oriented from $X$ to $Y$. Thus, if $D$ is a tournament, this means that every possible edge between $X$ and $Y$ is oriented from $X$ to $Y$. If $X=\{x\}$, then we simply write $x \rightarrow_{D} Y$ (and similarly if $Y=\{y\}$ ). We shall omit the subscript ' $D$ ' when the digraph is understood from context.

A set $X \subset V(D)$ is a cut-set if $D-X$ is not strongly-connected. A strongly-connected component in a digraph $D$ is a strongly-connected subdigraph of $D$ that is maximal with this property. Every digraph can be partitioned into its strongly-connected components, say $V(D)=$ $S_{1} \cup \cdots \cup S_{t}$ where $D\left[S_{i}\right]$ is strongly-connected for each $i \in[t]$ and $S_{i} \rightarrow S_{j}$ for all $1 \leq i<j \leq t$ (see Lemma 3.1). If $t \geq 2$, we then have $S:=\bigcup_{i<t} S_{i} \rightarrow S_{t}$. We call $S$ the source set and $S_{t}$ the sink set of $D$. Moreover, if $X=\left\{x_{1}, \ldots, x_{t}\right\} \subseteq V(D)$ is a transitive set with $x_{i} \rightarrow x_{j}$ for all $1 \leq i<j \leq t$, we refer to $x_{1}$ and $x_{t}$ as the source and sink of $X$, respectively.

Finally, given a positive integer $k$, a digraph is $k$-strongly-connected if it has at least $k+1$ vertices and if it remains strongly-connected upon the removal of any set of at most $k-1$ vertices.

\section{Preliminaries}

Here we collect some simple results that will be applied in several places throughout the paper. The first allows us to define source sets and sink sets of non-strongly-connected digraphs. The second allows us to assume that our digraphs are strongly-connected when considering dichromatic number.

Lemma 3.1. The vertex set of every digraph $D$ can be partitioned into nonempty sets $S_{1}, \ldots, S_{t}$ such that $D\left[S_{i}\right]$ is strongly-connected for every $i$, and $S_{i} \rightarrow S_{j}$ for all $1 \leq i<j \leq t$.

Proof. Say that two vertices $x, y$ are strongly-connected if there is a directed path from $x$ to $y$ and a directed path from $y$ to $x$ in $D$. This is clearly an equivalence relation, and we may take $S_{1}, \ldots, S_{t}$ to be the pairwise-disjoint equivalence classes. For the second claim, we may assume $t \geq 2$, since if $t=1$ it holds vacuously. Define a digraph $H$ whose vertex set is $[t]$ and we join $i$ to $j$ whenever there is an edge from $S_{i}$ to $S_{j}$ in $D$. If we cannot order the $S_{i}$ 's transitively as claimed, then $H$ contains a directed cycle $C=i_{1} i_{2} \ldots i_{k} i_{1}$ for some $k \geq 2$. But then $D\left[\bigcup_{j \in V(C)} S_{j}\right]$ is strongly-connected, which contradicts the maximality of $S_{1}, \ldots, S_{t}$.

Lemma 3.2. If $D$ is a digraph with $\chi(D) \geq r$, then $D$ contains a strongly-connected subdigraph $D^{\prime}$ with $\chi\left(D^{\prime}\right) \geq r$.

Proof. If $D$ itself is strongly-connected, then we are done. Otherwise, apply Lemma 3.1 and let $V(D)=S_{1} \cup \cdots \cup S_{t}$ be a partition of the vertex set of $D$ into strongly-connected components, such that $S_{i} \rightarrow S_{j}$ for all $1 \leq i<j \leq t$, and $t \geq 2$. Assume that we have $\chi\left(D\left[S_{i}\right]\right) \leq r-1$ for all $i \in[t]$. Colour the vertices in each $S_{i}$ with colours in $[r-1]$. Then this produces an $(r-1)$-colouring of $D$, a contradiction.

\section{CONSTRUCTIONS}

In this section, we examine the following constructions: a tournament $S_{r}$ with $\chi\left(S_{r}\right)=r$ and $\operatorname{sm}\left(S_{r}\right) \leq r-1$, a digraph $G_{r}$ with $\chi\left(G_{r}\right)=r$ and $\operatorname{sm}\left(G_{r}\right) \leq r-1$, and a digraph $D_{k}$ with out-degree $k$ and no strong $\vec{K}_{3}$ minor. 


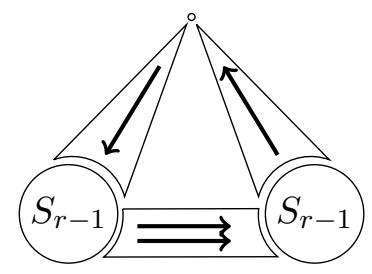

FiguRE 1. The tournament $S_{r}$

4.1. Digraphs with large dichromatic number and no large strong minor. Given digraphs $H, G, D$, let $\Delta(H, G, D)$ be the digraph obtained by taking vertex-disjoint copies of $H, G$, and $D$, adding all possible undirected edges with at most one endpoint in each of $V(H), V(G)$, and $V(D)$, and orienting the new edges so that $H \rightarrow G, G \rightarrow D$, and $D \rightarrow H$.

Construction 1. Let $S_{1}=K_{1}$ (i.e., the single vertex complete digraph with no edges). For $r \geq 2$ we set $S_{r}=\Delta\left(K_{1}, S_{r-1}, S_{r-1}\right)$.

We remark that this construction has appeared before in [2]. It is not difficult to check by induction that $\chi\left(S_{r}\right)=r$ for all $r \geq 1$. The following shows that, in general, dichromatic number $r$ is not sufficient to guarantee a strong $\vec{K}_{r}$ minor.

Proposition 4.1. For every $r \geq 2$ we have $\chi\left(S_{r}\right)=r$ and $\operatorname{sm}\left(S_{r}\right) \leq r-1$ for every $r \geq 2$.

Proof. As mentioned, $\chi\left(S_{r}\right)=r$ is easy to verify by induction on $r$. We turn to determining $\operatorname{sm}\left(S_{r}\right)$. Note that $S_{2}$ is simply a directed triangle and so clearly $\operatorname{sm}\left(S_{2}\right)=1$. Let $r \geq 3$ and suppose the result holds for smaller values. Since $S_{r}=\Delta\left(K_{1}, S_{r-1}, S_{r-1}\right)$ let us write $A$ and $B$ for the two copies of $S_{r-1}$, and $v$ for the copy of $K_{1}$. Clearly, at most one branch set from any strong minor in $S_{r}$ contains $v$. If $X \subseteq V\left(S_{r}\right)$ is a strongly-connected subset that does not contain $v$, then $X$ cannot contain vertices from both $A$ and $B$ (since $A \rightarrow B$ ). Similarly, there cannot be one branch set contained in $A$ and another contained in $B$. Since we can potentially add a new branch set in $S_{r}$ containing $v$ we have

$$
\operatorname{sm}\left(S_{r}\right) \leq \operatorname{sm}\left(S_{r-1}\right)+1 \leq r-1,
$$

as claimed.

Construction 2. Let $G_{1}=K_{1}$ and suppose $G_{r}$ has been constructed for some $r \geq 1$. To form $G_{r+1}$, consider first a transitive tournament $T_{r+1}$ on $r+1$ vertices. For each directed edge $e$ of $T_{r+1}$ add pairwise disjoint copies of $G_{r}$, denoted by $G_{r}^{e}$, such that $V\left(G_{r}^{e}\right) \cap V\left(T_{r+1}\right)=\varnothing$ for all e. Finally, for each directed edge $e=v w$ in $T_{r+1}$ and each vertex $u \in V\left(G_{r}^{e}\right)$ create a directed triangle uvw.

Proposition 4.2. For every $r \geq 2$ we have $\chi\left(G_{r}\right)=r$ and $\operatorname{sm}\left(G_{r}\right) \leq r-1$.

Proof. Note that $G_{2}$ is a directed triangle, so we have $\chi\left(G_{2}\right)=2$ and $\operatorname{sm}\left(G_{2}\right)=1$. So suppose $r \geq 3$ and the result holds for smaller values. Since each copy of $G_{r-1}$ in $G_{r}$ can be $(r-1)$ coloured, we can $r$-colour $G_{r}$ by using one extra colour for the transitive tournament. On the other hand, we cannot $(r-1)$-colour $G_{r}$ : assume there is an $(r-1)$-colouring of $G_{r}$, then some two vertices $x, y \in V\left(T_{r}\right)$ receive the same colour, say 1 . Let $e=x y$ be the corresponding edge in $T_{r}$. As $\chi\left(G_{r-1}^{e}\right)=r-1$, the colour 1 must appear in $G_{r-1}^{e}$, and so we obtain a directed triangle in colour 1 , a contradiction.

Next, we shall show that $\operatorname{sm}\left(G_{r}\right) \leq r-1$. Let $t=\operatorname{sm}\left(G_{r}\right)$. Consider a strong $\vec{K}_{t}$ minor in $G_{r}$ with branch sets $B_{1}, \ldots, B_{t}$. Since $T_{r}$ is not strongly-connected, $B_{i} \not \subset V\left(T_{r}\right)$ for each $i=1, \ldots, t$. Observe also that if for some edge $e=x y$ of $T_{r}, B_{i} \cap V\left(G_{r-1}^{e}\right) \neq \emptyset$ and $B_{i} \backslash V\left(G_{r-1}^{e}\right) \neq \emptyset$, then $B_{i}$ contains both $x$ and $y$; otherwise, $B_{i}$ does not induce a strongly-connected digraph. There are two cases to consider.

Case 1. There is $i \in[t]$ such that $B_{i} \subseteq V\left(G_{r-1}^{e}\right)$ for some edge $e=x y$ of $T_{r}$.

Without loss of generality, assume $i=t$. Let $V^{\prime}=V\left(G_{r-1}^{e}\right)$. Then any other $B_{j}, j=1, \ldots, t-1$ should either be contained in $V^{\prime}$ or contain $x$ or $y$. If all $B_{i}$ 's are contained in $V^{\prime}$, then by 
induction $t \leq r-2$. Thus, we can assume that there is $j \in\{1, \ldots, t-1\}$ such that $B_{j}$ contains $x$ or $y$. If $B_{j}$ intersects $V^{\prime}$, we have that $B_{j}$ contains both $x$ and $y$ by an observation before the statement of the case. If $B_{j}$ does not contain vertices from $V^{\prime}$ it also must contain both $x$ and $y$, otherwise the edges between $B_{t}$ and $B_{j}$ go in one direction only. Since the $B_{i}$ 's are pairwise disjoint, there is exactly one such index $j$, such that $B_{j}$ is not contained in $V^{\prime}$. The number of branch sets from $B_{1}, \ldots, B_{t}$ that are contained in $V^{\prime}$ is by induction at most $r-2$. Thus $\operatorname{sm}\left(G_{r}\right)=t \leq r-2+1=r-1$.

Case 2. For each branch set $B_{i}$ there exists an edge e of $T_{r}$ such that $B_{i}$ contains both vertices of $e$.

Since the $B_{i}$ 's are pairwise disjoint, the respective edges $e$ must also be pairwise disjoint. Since $T_{r}$ has at most $r / 2 \leq r-1$ pairwise disjoint edges, $t \leq r-1$. This completes the second case, and thus the proof.

4.2. Digraphs with large out-degree and no large strong minor. Here we show that large out-degree is not sufficient to guarantee strong $\vec{K}_{r}$ minors in general digraphs, and thus prove Theorem 1.4. To this end, we need Thomassen's [23] construction of digraphs with large out-degree and no even cycle. The construction proceeds as follows.

Construction 3. Let $D_{1}$ be a directed triangle. Suppose $D_{k}$ is defined by induction. Now, add pairwise disjoint sets $A_{x} \cup\left\{x^{\prime}\right\}$ where $\left|A_{x}\right|=k+1$, for every vertex $x \in V\left(D_{k}\right)$, all of them disjoint from $V\left(D_{k}\right)$. We then add all directed edges between $A_{x}$ and $N_{D_{k}}^{+}(x) \cup\{x\}$ such that $A_{x} \rightarrow\{x\} \cup N_{D_{k}}^{+}(x)$, and $x \rightarrow x^{\prime}$. Finally, add all edges from $x^{\prime}$ to $A_{x}$, and denote by $D_{k+1}$ the resulting digraph.

Then $D_{k}$ clearly has minimum out-degree $k$, and one can check that it contains no even directed cycle (in particular, it contains no pair of vertices $x, y$ with $x y, y x \in E\left(D_{k+1}\right)$ ). The following asserts that $D_{k}$ does not contain large strong clique minors. In particular, large out-degree alone is not sufficient to guarantee strong minors in digraphs.
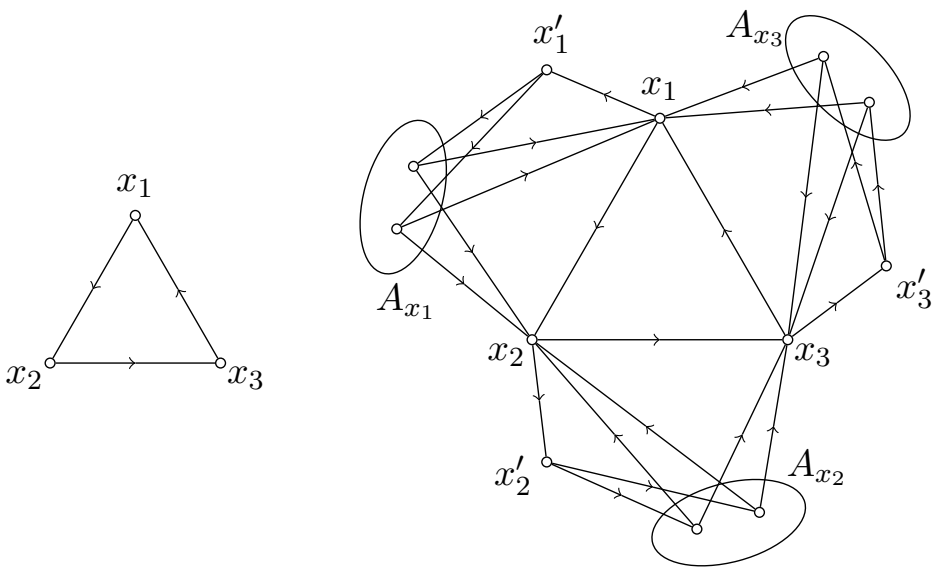

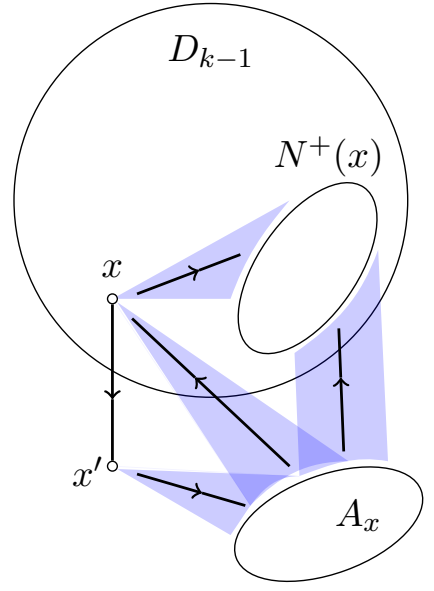

Figure 2. The digraph $D_{k}$ for $k=1,2$ and sketch for general $k$

Proposition 4.3. For every $k \geq 1, D_{k}$ does not contain a strong $\vec{K}_{3}$ minor.

Proof. We proceed by induction on $k$. This is clearly true for $k=1$, so suppose $k \geq 2$ and the result holds for smaller values of $k$. Suppose, by way of contradiction, that $D_{k+1}$ contains a strong $\vec{K}_{3}$ minor with branch sets $U, W, X$. Since $D_{k}$ contains no strong $\vec{K}_{3}$ minor, it must be the case that at least one of these branch sets intersects $A_{x} \cup\left\{x^{\prime}\right\}$ for some $x \in V\left(D_{k}\right)$. So suppose $U$ intersects $A_{x} \cup\left\{x^{\prime}\right\}$. We claim that $x \in U$.

Indeed, first suppose that $x^{\prime} \in U$. Since $N^{-}\left(x^{\prime}\right)=\{x\}$, any nontrivial directed path to $x^{\prime}$ passes through $x$. As $D[U]$ is strongly-connected, we either have $x \in U$ or $U=\left\{x^{\prime}\right\}$. The latter cannot hold, since otherwise we must have both $x \in X$ and $x \in W$, a contradiction. Thus, we have $x \in U$. 
Now suppose that $y \in U$ for some $y \in A_{x}$. Then since $N^{-}(y)=\left\{x^{\prime}\right\}$, any nontrivial directed path to $y$ includes $x^{\prime}$, so either $x^{\prime} \in U$ or $U=\{y\}$. In the latter case we must have both $x^{\prime} \in X$ and $x^{\prime} \in W$, a contradiction. In the former case we have $x \in U$, by the above argument.

In any case, $x \in U$ as claimed. But then $U^{\prime}=U \cap V\left(D_{k}\right)$ induces a strongly-connected subdigraph in $D_{k}$. Indeed, if $U^{\prime}=\{x\}$, then it is trivially strongly-connected in $D_{k}$. Otherwise, let $u, v \in U^{\prime}$ be distinct vertices. Then any path from $u$ to $v$ that uses vertices not in $D_{k}$, say from $A_{x} \cup\left\{x^{\prime}\right\}$, passes through $x$ and eventually an out-neighbour $z$ of $x$. So there is a path from $u$ to $v$ contained in $U^{\prime}$, obtained by going from $x$ directly to $z$ and continuing outside of $A_{x} \cup\left\{x^{\prime}\right\}$. Similarly, we have that $W^{\prime}=W \cap V\left(D_{k}\right)$ and $X^{\prime}=X \cap V\left(D_{k}\right)$ induce strongly-connected subdigraphs in $D_{k}$.

Since $U, W$, and $X$ are pairwise disjoint, then for any $x$ at most one of these three sets intersects $A_{x} \cup\left\{x^{\prime}\right\}$. Since there are no edges between $A_{x} \cup\left\{x^{\prime}\right\}$ and $A_{y} \cup\left\{y^{\prime}\right\}$ for distinct $x$ and $y$, all edges between $U, W$, and $X$ are the edges between $U^{\prime}, W^{\prime}$, and $X^{\prime}$. Thus $U^{\prime}, W^{\prime}$, and $X^{\prime}$ form branch sets of a strong $\vec{K}_{3}$ minor in $D_{k}$, a contradiction.

\section{STRONG MINORS IN TOURNAMENTS}

5.1. Large dichromatic number in tournaments. The aim of this section is to prove Theorem 1.2. Before doing so, however, we shall prove the following warm-up result, which shows that dichromatic number linear in $r$ is sufficient to guarantee strong $\vec{K}_{r}$ minors in tournaments.

Proposition 5.1. Let $r \geq 1$ be an integer and suppose $T$ is a tournament with $\chi(T) \geq 3 r$. Then $\operatorname{sm}(T) \geq r$.

Proof. We proceed by induction on $r$. Clearly, this is true for $r=1$, so we may assume $r \geq 2$ and the result holds for smaller values of $r$. Suppose $T$ is a tournament with $\chi(T) \geq 3 r$. We may assume $T$ is strongly-connected; otherwise, by Lemma 3.2, we can pass to a strongly-connected component with dichromatic number at least $3 r$. Let $A \subset V(T)$ be a largest transitive subset with source $s$ and sink $t$. Since $T$ is strongly-connected there exists a directed path in $T$ from $t$ to $s$. Pick a shortest directed path $P$ from $t$ to $s$. Note that $\chi(T[V(P)]) \leq 2$ : by the minimality of $P$, all edges between vertices of $P$ that have distance at least 2 on the path are oriented backwards. It follows that vertices at odd/even distances from the initial vertex produce a 2 -colouring of $P$. Then $\chi(T[A \cup V(P)]) \leq 3$. Let $T^{\prime}=T-(A \cup V(P))$. It follows that $\chi\left(T^{\prime}\right) \geq 3 r-3=3(r-1)$. By induction, we have that $T^{\prime}$ contains a strong $\vec{K}_{r-1}$ minor.

Observe that the maximality of $A$ implies that for every vertex $v \in V(T) \backslash A$, $v$ has at least one out-neighbour and at least one in-neighbour in $A$. In particular, for each branch set of our strong $\vec{K}_{r-1}$ minor in $T^{\prime}$, there are edges in both directions between $A$ and this branch set. Finally, note that $T[A \cup V(P)]$ is strongly-connected, so $A \cup V(P)$ can be taken as the $r$ th branch set in a strong $\vec{K}_{r}$ minor in $T$, completing the induction and the proof.

The proof of Proposition 5.1 follows by first considering a maximal transitive set, ensuring that this set is in and out 'dominating', and then making this set strongly-connected by attaching a shortest path. Using this naive approach, we can only guarantee the the resulting set has dichromatic number at most 3 . We would like to reduce ' 3 ' to ' 2 ', and thus in order to prove Theorem 1.2, we must work a little harder. The following lemma, which may be viewed as the heart of the proof of Theorem 1.2, shows that one can find strongly-connected dominating sets of dichromatic number 2 in any strongly-connected tournament. This is clearly tight on the dichromatic number and it is a result of independent interest.

Before embarking on the proof, let us introduce some useful terminology. Let $D$ be a digraph, let $S \subseteq V(D)$, and let $X \subseteq V(D) \backslash S$. We say that $S$ out-dominates (in-dominates, resp.) $X$ if every $x \in X$ has at least one in-neighbour (out-neighbour, resp.) in $S$. If $X=V(D) \backslash S$, we say that $S$ is out(in)-dominating. If $S$ is both out-dominating and in-dominating, we shall say that $S$ is dominating (or, that it is a dominating set).

Lemma 5.2. Let $T$ be a strongly-connected tournament. Then there exists a subset $R \subseteq V(T)$ satisfying the following: 
- $T[R]$ is strongly-connected,

- $R$ is dominating, and

- $\chi(T[R])=2$.

Proof. We illustrate the proof in Figure 3. Let $x$ be a vertex in $T$ with maximum out-degree in $T$. Let $S(x)$ be a largest possible transitive subtournament with sink $x$. Denote by $y$ the source of $S(x)$. Observe that since $S(x)$ is maximum-sized, it must be out-dominating: for all $v \in V(T) \backslash S(x)$ there is $u \in S(x)$ such that $u \rightarrow v$. Moreover, as $x$ has maximum out-degree in $T$, there exists a vertex $w^{*} \in V(T) \backslash S(x)$ such that $x \rightarrow w^{*}$ and $w^{*} \rightarrow y$, otherwise $y$ would have a larger out-degree than $x$.

Now, let $F=\bigcap_{s \in S(x)} N^{+}(s)$. The set $F$ consists of the vertices that are not in-dominated by $S(x)$. If $F=\varnothing$, then we are done, since then we may take $R=S(x) \cup\left\{w^{*}\right\}$. So we may assume that $F \neq \varnothing$. Let $F^{+} \subseteq F$ be the set of vertices in $F$ that have an out-neighbour in $V(T) \backslash F$. Since $T$ is strongly-connected, it follows that $F^{+} \neq \varnothing$. We need to find a set (hopefully of small dichromatic number) that in-dominates the vertices in $F$. First, we prove the following claim showing that we may partition $F$ in a useful way.

Claim 1. For some $k \geq 1$ there is a partition $F=F_{1} \cup \cdots \cup F_{k}$ such that $F_{1}=F^{+}$and for every $1 \leq i \leq k-1$

(1) for every $u \in F_{i+1}$ there is $z \in F_{i}$ such that $u \rightarrow z$, and

(2) for every $j, j>i+1, F_{i} \rightarrow F_{j}$.

Proof. Let $F_{1}=F^{+}$. Suppose $F_{1}, \ldots, F_{i}$ have been constructed for some $i \geq 1$. Let $Y=$ $F \backslash\left(\bigcup_{j \in[i]} F_{j}\right)$. If $Y=\varnothing$, then stop with $k:=i$. Otherwise, let

$$
F_{i+1}=\left(\bigcup_{u \in F_{i}} N^{-}(u)\right) \cap Y .
$$

Note that $F_{i+1}$ is nonempty due to the strong-connectivity of $T$. Indeed, if $F_{i+1}=\varnothing$, then $F_{i} \rightarrow Y$ and since $Y \cap F_{1}=\varnothing$, no vertex of $Y$ has an out-neighbour in $V(T) \backslash F$. Thus, there is no directed path from a vertex in $Y$ to a vertex in $F_{1}=F^{+}$, a contradiction to the strong-connectivity assumption.

Also, note that

$$
\bigcup_{j \in[i]} F_{j} \rightarrow Y \backslash F_{i+1}
$$

By construction, every vertex in $F_{i+1}$ has at least one out-neighbour in $F_{i}$. This completes the proof of the claim.

Let $S_{k}$ be a largest transitive set contained in $T\left[F_{k}\right]$. If $F_{k-1}$ exists (i.e. $k \geq 2$ ), let $S=$ $S_{k} \cup S_{k-1}$, where $S_{k-1} \subseteq F_{k-1}$ is as large as possible such that $S$ is transitive, and all vertices of $S_{k-1}$ lie after the vertices in $S_{k}$ in the transitive order. If $k=1$, let $S=S_{1}$. Let $s$ denote the sink of $S$. In particular, either $s \in F_{k}$ or $s \in F_{k-1}$. Intuitively, $S$ in-dominates $F$ (which we shall show later), and it remains to 'connect' $S$ to $S(x)$ to find a dominating set that is strongly-connected.

To this end, consider a shortest directed path $P=s \ldots x_{1}$ contained in $F$ from $s$ to a vertex in $F_{1}$; such a path exists by Claim 1 . Also, observe that since $P$ is a shortest path in $F$ to $F_{1}$, it has exactly one vertex in each $F_{i}$. Now, since $x_{1} \in F_{1}=F^{+}$, by definition of $F^{+}$we may choose an out-neighbour $w \in V(T) \backslash(F \cup S(x))$ of $x_{1}$. It is possible that $w=w^{*}$, but this only makes the proof simpler. So we assume that $w \neq w^{*}$. Finally, let

$$
R=S(x) \cup S \cup V(P) \cup W .
$$

The following sequence of claims shows that $R$ has all of the desired properties (see Figure 3 ).

Claim 2. $T[R]$ is strongly-connected.

Proof. First, note that $T[S(x) \cup W]$ is strongly-connected. Indeed, $T\left[S(x) \cup\left\{w^{*}\right\}\right]$ is stronglyconnected since $w^{*} \in N^{-}(y) \cap N^{+}(x)$. As $w \notin F$, it has an out-neighbour $v \in S(x)$, and 
the maximality of $S(x)$ implies that $w$ has an in-neighbour $v^{\prime} \in S(x)$. It follows that $T[R]$ is strongly-connected, as claimed.

Claim 3. $R$ is a dominating set in $T$.

Proof. $S(x)$ dominates $V(T) \backslash(S(x) \cup F)$ : the maximality of $S(x)$ implies that it is outdominating, and it additionally in-dominates $V(T) \backslash F$ (since $F$ is exactly the set of vertices not in-dominated by $S(x))$. So it suffices to show that $R$ in-dominates $F \backslash(S \cup V(P))$. In particular, we claim that $S$ in-dominates $F \backslash(S \cup V(P))$. Indeed, by (2) of Claim 1 we have

$$
\bigcup_{i \leq k-2} F_{i} \rightarrow S \cap F_{k}
$$

so it suffices to show that $S$ in-dominates $\left(F_{k} \cup F_{k-1)}\right) \backslash S$. Recall that $S=S_{k} \cup S_{k-1}$ where $S_{k}$ is a largest transitive set in $F_{k}$, and $S_{k-1}$ is a largest possible transitive extension of $S_{k}$ in $F_{k-1}$ such that all vertices of $S_{k-1}$ lie after $S_{k}$ in the transitive order. Thus, if there is some vertex $z \in\left(F_{k} \cup F_{k-1}\right) \backslash S$ such that $S \rightarrow z$, we obtain a contradiction with our choice of $S$. It follows that $S$ in-dominates $\left(F_{k} \cup F_{k-1}\right) \backslash S$, as claimed.

Claim 4. $\chi(T[R])=2$.

Proof. We produce a 2-colouring of $R$ that depends on the parity of $k$ and also on whether $s$ (the sink of $S$ ) is in $F_{k}$ or $F_{k-1}$. We deal with the case when $k$ is even first, and sketch the proof when $k$ is odd, as the proof is nearly identical. Recall that as $P=s \ldots x_{1}$ is a shortest $s-F_{1}$ path in $F$, it has exactly one vertex in each $F_{i}$ (except for maybe $F_{k}$ ).

Case 1: $k$ is even. First, suppose that $k=2$. If $s \in F_{2}$, then let one colour class be $S(x) \cup\left\{x_{1}\right\}$ and the other $S \cup W$. Note that the latter set is transitive since $S \subseteq F_{2}$ and so sends no out-edges to $V(T) \backslash F$. On the other hand, if $s \in F_{1}$, then let one colour class be $S(x) \cup\left(S \cap F_{1}\right)$ and the other $\left(S \cap F_{2}\right) \cup W$.

Now, suppose $k>2$ and $x_{k}:=s \in F_{k}$. Write $P=x_{k} x_{k-1} \ldots x_{1}$ with $x_{i} \in F_{i}$ for all $i \in[k]$. Let $P_{\text {odd }}=\left\{x_{1}, x_{3}, \ldots, x_{k-1}\right\}$ and $P_{\text {even }}=\left\{x_{2}, x_{4}, \ldots, x_{k}\right\}$. Then we can let one colour class be $S(x) \cup P_{\text {odd }}$ and the other $S \cup P_{\text {even }} \cup W$. Indeed, $S(x)$ is transitive and $S(x) \rightarrow P_{\text {odd }}$. Moreover, $T\left[P_{\text {odd }}\right]$ is transitive by (2) of Claim 1. Similarly, since $x_{k}=s \in F_{k}$ and $k>2$, we have $S \cup P_{\text {even }} \subseteq F \backslash F_{1}$ and so $W \rightarrow S \cup P_{\text {even. }}$. As before, $T\left[S \cup P_{\text {even }}\right]$ is transitive and clearly so is $W$.

Lastly, we consider the case when $x_{k-1}:=s \in F_{k-1}$, i.e., when $P=x_{k-1}, \ldots, x_{1}$ let $P_{\text {even }}=$ $\left\{x_{2}, \ldots, x_{k-2}\right\}$ and let $P_{\text {odd }}=V(P) \backslash P_{\text {even }}$. In this case, we let one colour class be $S(x) \cup P_{\text {odd }} \cup$ $\left(S \cap F_{k-1}\right)$ and the other be $\left(S \cap F_{k}\right) \cup P_{\text {even }} \cup W$. It is routine to check that this is indeed a 2-colouring using the same arguments as above.

Case 2: $k$ is odd. If $k=1$ let $S(x) \cup S$ be one colour class, and $W$ the other. If $k>1$, then an analogous argument as in the previous case yields a 2-colouring of $R$.

Hence, $R$ satisfies all of the claimed properties. This completes the proof of the lemma. 


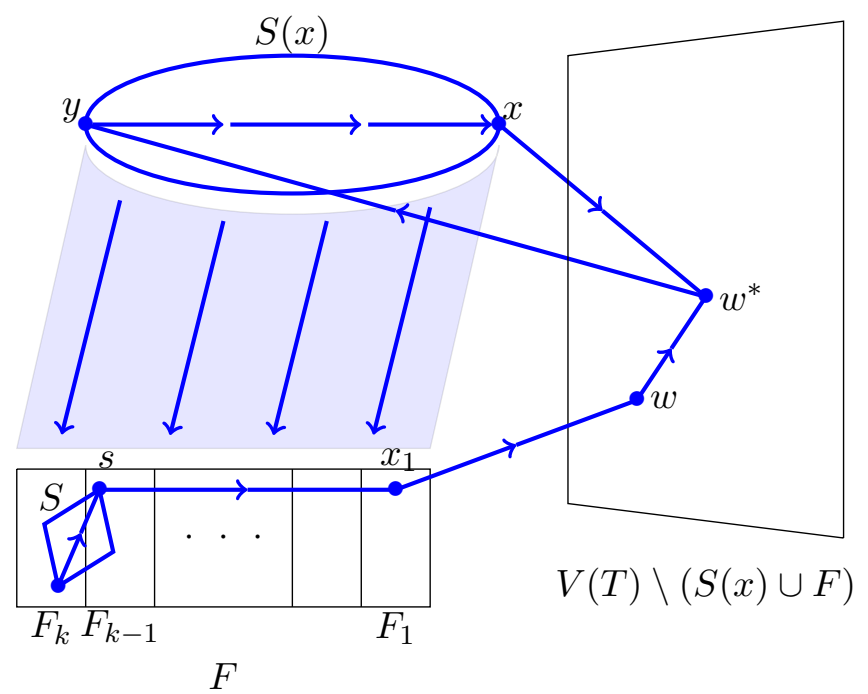

FiguRE 3. The proof of Lemma 5.2. The direction of $w w^{*}$ is not important for the proof.

We can now easily deduce Theorem 1.2.

Proof of Theorem 1.2. We have already established the second part of the statement in Proposition 4.1. It remains to prove the first part.

We proceed by induction on $r$. Clearly, the result holds for $r=1$, so we suppose $r \geq 2$ and the theorem holds for smaller values. Let $T$ be a tournament with $\chi(T) \geq 2 r$, and, by Lemma 3.2, we may assume $T$ is strongly-connected. Lemma 5.2 grants a subset $R \subseteq V(T)$ such that $T[R]$ is strongly-connected, $R$ is dominating in $T$, and $\chi(T[R])=2$. Letting $T^{\prime}=T-R$, we see that $\chi\left(T^{\prime}\right) \geq 2 r-2=2(r-1)$, and so by induction $T^{\prime}$ contains a strong $\vec{K}_{r-1}$ minor. Taking $R$ as the $r$ th branch set yields a strong $\vec{K}_{r}$ minor in $T$.

Perhaps the correct constant in Theorem 1.2 should be ' 1 ' instead of ' 2 ' as indicated by the construction $S_{r}$. In other words, it is possible that any tournament with dichromatic number at least $r+1$ contains a strong $\vec{K}_{r}$ minor. We can prove this in the first nontrivial case:

Proposition 5.3. If $T$ is a tournament with $\chi(T) \geq 3$, then $\operatorname{sm}(T) \geq 2$.

Proof. Pick a directed triangle $C_{1}$ in $T$. Since $\chi(T) \geq 3, T-C_{1}$ is nonempty. If there is a vertex $v \notin C_{1}$ that sends edges in both directions to $C_{1}$, then we are done. Otherwise, $T-C_{1}$ can be partitioned into two sets $A_{1}$ and $B_{1}$ such that $A_{1} \rightarrow C_{1}$ and $C_{1} \rightarrow B_{1}$. If there is an edge $e$ from $B_{1}$ to $A_{1}$, then we are done. Indeed, one branch set is the directed triangle formed from $e$ and one vertex of $C_{1}$, and the other branch set is just one of the remaining vertices of $C_{1}$. So we may assume that $A_{1} \rightarrow B_{1}$. In this case, we either have $\chi\left(A_{1}\right) \geq 3$ or $\chi\left(B_{1}\right) \geq 3$. Without loss of generality, $\chi\left(A_{1}\right) \geq 3$, and so we pass to this subtournament and repeat the argument.

In this process we obtain a sequence of nonempty sets strictly decreasing in size, so it must eventually terminate with a strong $\vec{K}_{2}$ minor.

5.2. Large out-degree in tournaments. In this section we turn our attention to investigating the presence of strong minors in tournaments with a minimum out-degree condition. In particular, we prove Theorem 1.3. The strategy of our proof is as follows. First, we show that there is a constant $C^{\prime}$ such that any tournament that is $C^{\prime} r \sqrt{\log r}$-strongly-connected contains a strong $\vec{K}_{r}$ minor (see Lemma 5.8). Given a tournament $T$ with $\delta^{+}(T) \geq C r \sqrt{\log r}$ where $C \gg C^{\prime}$, we may assume that no subtournament of $T$ is $C^{\prime} r \sqrt{\log r}$-strongly-connected. This assumption together with the minimum out-degree condition allows us to iteratively construct a strong $\vec{K}_{r}$ minor in which every branch set is, in fact, a directed triangle. This last step is made precise in Lemma 5.9 and Lemma 5.10. 
Before proceeding, we need a few preliminary results. Given a positive integer $k$ and a digraph $D$, we say that $D$ is $k$-linked if $|V(D)| \geq 2 k$ and for any two disjoint sets of vertices $\left\{x_{1}, \ldots, x_{k}\right\}$ and $\left\{y_{1}, \ldots, y_{k}\right\}$ of $k$ vertices each, there are pairwise vertex disjoint directed paths $P_{1}, \ldots, P_{k}$ such that $P_{i}$ has initial vertex $x_{i}$ and terminal vertex $y_{i}$ for every $i \in[k]$. We shall use the following theorem of Pokrovskiy [18], showing that large enough strong-connectivity guarantees linkedness in tournaments.

Theorem 5.4 (Pokrovskiy [18]). For every integer $k \geq 1$, any $452 k$-strongly-connected tournament is k-linked.

Recall that a weak minor has the same definition as a strong minor except that we only require that the branch sets induce connected (not necessarily strongly-connected) subgraphs. As mentioned in the Introduction, Jagger [11] investigated average degree conditions for finding weak minors in digraphs. In the context of tournaments, we need the following:

Theorem 5.5 (Jagger [11]). There exists an absolute constant $C>0$ such that the following holds. If $r$ is a positive integer and $T$ is a tournament with at least $C r \sqrt{\log r}$ vertices, then $T$ contains a weak $\vec{K}_{r}$ minor.

During the course of the proof of Theorem 1.3, we shall construct an auxiliary (undirected) graph and apply the following classical result:

Theorem 5.6 (Kostochka [15], Thomason [22]). There exists a constant $c>0$ such that for every positive integer $r$, every graph of average degree at least $\mathrm{cr} \sqrt{\log r}$ contains a $K_{r}$ minor.

Finally, we need one more preliminary result. We say that a subset $B \subset V(T)$ is $K$-nearlyregular if either $d_{T}^{-}(v) \leq d_{T}^{+}(v) \leq K d_{T}^{-}(v)$ for every $v \in B$, or $d_{T}^{+}(v) \leq d_{T}^{-}(v) \leq K d_{T}^{+}(v)$ for every $v \in B$. The following lemma appears in [5]. We reproduce the proof here for convenience.

Lemma 5.7. Any tournament $T$ contains a 4-nearly-regular subset of vertices $R$ of size at least $\lfloor|T| / 20\rfloor$. In particular, any $v \in R$ has in and out-degree at least $|T| / 6$.

Proof. Let $|T|=n$; we may assume $n \geq 20$, otherwise the result is trivial. Let $R \subset V(T)$ be the set of vertices for which either the ratio between the out-degree and in-degree or vice-versa is between 1 and 4 . If $|R| \geq n / 10$, then we are done, as we may pass to a subset $A \subset R$ of at least half the size of $R$ that is 4-nearly-regular. If $|R|<n / 10$, then let $T^{\prime}=T-R$, so that $\left|T^{\prime}\right|>9 n / 10$. Let $T_{1}^{\prime}$ be the set of vertices $v \in V\left(T^{\prime}\right)$ for which $d_{T}^{+}(v)>4 d_{T}^{-}(v)$ and $T_{2}^{\prime}$ be those vertices $v \in V\left(T^{\prime}\right)$ for which $d_{T}^{-}(v)>4 d_{T}^{+}(v)$. Suppose without loss of generality that $\left|T_{1}^{\prime}\right| \geq\left|T_{2}^{\prime}\right|$, so that $\left|T_{1}^{\prime}\right| \geq\lceil 9 n / 20\rceil$. Since each tournament on $t$ vertices has a vertex of in-degree $\geq\lceil(t-1) / 2\rceil$, this implies that there is a vertex $u$ in $T_{1}^{\prime}$ which has in-degree inside $T_{1}^{\prime}$ at least $(\lceil 9 n / 20\rceil-1) / 2$. However,

$$
d_{T}^{-}(u)<\frac{1}{4} d_{T}^{+}(u)<\frac{1}{4}\left(n-d_{T}^{-}(u)\right),
$$

implying that $d_{T}^{-}(u)<n / 5$. Thus, we obtain

$$
(\lceil 9 n / 20\rceil-1) / 2 \leq d_{T}^{-}(u)<n / 5,
$$

which yields a contradiction for $n \geq 20$.

Finally, let $R \subseteq V(T)$ be 4-nearly-regular, and assume without loss of generality that $d_{T}^{-}(u) \leq$ $d_{T}^{+}(u) \leq 4 d_{T}^{-}(u)$ for all $u \in R$. If for some $v \in R$ we have $d_{T}^{-}(v)<n / 6$, then $d_{T}^{+}(v)<2 n / 3$, and so $n-1=d_{T}^{+}(u)+d_{T}^{-}(u)<5 n / 6$, a contradiction since $n \geq 20$.

With these preliminaries in place, we shall prove our first lemma, guaranteeing strong $\vec{K}_{r}$ minors in $C^{\prime} r \sqrt{\log r}$-strongly-connected tournaments. The strategy is to first embed a weak $\vec{K}_{r}$ minor in a 'nice' portion of the tournament (using Theorem 5.5), and then make each branch set strongly-connected by linking appropriate pairs of vertices.

Lemma 5.8. Let $r$ be a positive integer. There exists a constant $C^{\prime}>0$ such that if $T$ is a $C^{\prime} r \sqrt{\log r}$-strongly-connected tournament, then $\operatorname{sm}(T) \geq r$. 
Proof. Put $C_{0}=\max \{C, 24\}$ where $C$ is given by Theorem 5.5. Let $C^{\prime}=40 C_{0}$, and suppose $T$ is a tournament that is $C^{\prime} r \sqrt{\log r}$-strongly-connected. By Lemma 5.7 we can find a subset $S \subset V(T)$ of size at least $\lfloor|T| / 20\rfloor \geq C_{0} r \sqrt{\log r}$ such that all vertices of $S$ have in and out degree at least $|T| / 6$. Take a subset $S^{\prime} \subset S$ of size exactly $C_{0} r \sqrt{\log r}$ (ignoring floors/ceilings) and let $T^{\prime}=T-S^{\prime}$. Find a weak $\vec{K}_{r}$ minor in $S^{\prime}$ with branch sets $B_{1}, \ldots, B_{r}$, using Theorem 5.5.

Since $T^{\prime}$ is still $19 C_{0} r \sqrt{\log r}$-strongly-connected, by Theorem $5.4, T^{\prime}$ is $\left(19 C_{0} / 452\right) r \sqrt{\log r}$ linked. Hence, $T^{\prime}$ is $r$-linked by our choice of $C_{0}$. We now construct a strong $\vec{K}_{r}$ minor as follows. For each $i \in[r]$, if $T\left[B_{i}\right]$ is already strongly-connected, then we take $B_{i}$ as a branch set. Otherwise, $B_{i}$ decomposes into strongly-connected components $X_{1}^{i}, \ldots, X_{t}^{i}$ for some $t \geq 2$, such that $X_{p}^{i} \rightarrow X_{q}^{i}$ for all $1 \leq p<q \leq t$. Let $s_{i}^{1}$ be any vertex in $X_{1}^{i}$ and let $s_{i}^{2}$ be any vertex of $X_{t}^{i}$.

Now, every vertex in $S^{\prime}$ has many in and out-neighbours in $T^{\prime}$. More precisely, every vertex in $S^{\prime}$ has at least

$$
|T| / 6-\left|S^{\prime}\right| \geq \frac{20 C_{0} r \sqrt{\log r}}{6}-C_{0} r \sqrt{\log r}>C_{0} r \sqrt{\log r}
$$

in-neighbours and out-neighbours in $T^{\prime}$. Thus, for each $s_{i}^{1}$ we may choose an in-neighbour $u_{i}^{1} \in V\left(T^{\prime}\right)$, and for each $s_{i}^{2}$ we may choose an out-neighbour $u_{i}^{2} \in V\left(T^{\prime}\right)$, such that the vertices $u_{i}^{j}$ are all pairwise distinct. In this way, we have disjoint sets $X=\left\{u_{1}^{1}, \ldots, u_{s}^{1}\right\}$ and $Y=\left\{u_{1}^{2}, \ldots, u_{s}^{2}\right\}$ where $s \leq r$. Finally, use the fact that $T^{\prime}$ is $r$-linked to find pairwise vertexdisjoint directed paths $P_{i}$ in $T^{\prime}$ from $u_{i}^{1}$ to $u_{i}^{2}$ for each $i \in[s]$. Clearly, $T\left[B_{i} \cup V\left(P_{i}\right)\right]$ is strongly-connected for each $i \in[s]$, and thus we have found a strong $\vec{K}_{r}$ minor in $T$.

Lemma 5.8 is tight up to the constant $C$, by considering a random tournament $T$ on $c r \sqrt{\log r}$ vertices. As mentioned in the Introduction, following the argument of Bollobás, Catlin, and Erdős [3], w.h.p. it contains no strong $\vec{K}_{r}$ minor (in fact, it contains no $r$ pairwise disjoint nonempty sets with edges in each direction between each pair of sets). Additionally, it is not hard to show that w.h.p. $T$ is $(c r \sqrt{\log r}) / 10$-strongly-connected. This can be seen by noting that w.h.p. every two vertices have at least approximately $|T| / 10$ directed paths of length 2 in each direction.

The following two lemmas allow us to find some structure in a tournament with large minimum out-degree, under the additional assumption that it is not highly strongly-connected. We use the following notation in their formulations. If $\mathcal{H}$ is a family of subdigraphs of $D$, let $\bigcup \mathcal{H}$ denote the set $\bigcup_{H \in \mathcal{H}} V(H)$.

Lemma 5.9. Let $m \geq 2$ and $d \geq 1$ be integers and suppose $T$ is a vertex-minimal tournament with the property that $\delta^{+}(T) \geq d$. Then there exists a positive integer $t \leq m-1$ such that the following holds. If $T$ is not $m$-strongly-connected, then we can find a non-empty set $S=$ $\left\{w_{1} v_{1}, \ldots, w_{t} v_{t}\right\}$ of pairwise vertex-disjoint directed edges and a subtournament $T^{\prime} \subseteq T$ with the following properties:

- $V\left(T^{\prime}\right) \cap(\bigcup S)=\varnothing$.

- For any $i \in[t]$ we have $\left|N^{+}\left(v_{i}\right) \cap T^{\prime}\right| \geq\left|T^{\prime}\right|-m+1$, and $\left|N^{-}\left(w_{i}\right) \cap T^{\prime}\right| \geq\left|T^{\prime}\right|-d+1$.

- $\delta^{+}\left(T^{\prime}\right) \geq d-2 t$.

Proof. Assume that $T$ is not $m$-strongly-connected and let $R$ be a smallest cut-set of size at most $m-1$. Observe that $R \neq \varnothing$ : if $T$ itself is not strongly-connected, then consider the source set $X$ and sink set $Y$ of $T$. Since $X \rightarrow Y$, we must have $\delta^{+}(T[Y]) \geq d$, contradicting the minimality of $T$.

Let $A$ and $B$ denote the source set and sink set of $T-R$, respectively, and define

$$
R^{\prime}=\left\{x \in R:\left|N^{+}(x) \cap(B \cup R)\right|<d\right\} .
$$

The minimality of $T$ implies that $R^{\prime} \neq \varnothing$ : if $R^{\prime}=\varnothing$, then $\delta^{+}(T[B \cup R]) \geq d$, a contradiction. Now, choose a maximum matching $M=\left\{w_{1} v_{1}, \ldots, w_{t} v_{t}\right\}$ from $R^{\prime}$ to $A$ and let $T^{\prime}=T[(B \cup$ $R) \backslash V(M)]$ and $S=M$. We claim that $T^{\prime}$ and $S$ satisfy the required properties. Clearly, the first property is satisfied. To see the last property suppose that $x \in B \cup\left(R \backslash R^{\prime}\right)$, then its degree is at least $d-t$ in $T^{\prime}$. On the other hand, if $x \in R^{\prime} \backslash V(M)$, since we can not extend 
the matching $M, N_{T}^{+}(x) \cap A \subset V(M)$, which implies $d_{T^{\prime}}^{+}(x) \geq d-|V(M)|=d-2 t$. To see the second property, consider a vertex $v_{i}$. Then $v_{i} \rightarrow B$, and so there are at most $|R| \leq m-1$ vertices in $\left|T^{\prime}\right|$ that are in-neighbours of $v_{i}$. Similarly, consider a vertex $w_{i}$. Since $w_{i} \in R^{\prime}$, by definition $\left|N^{+}(x) \cap(B \cup R)\right|<d$. Thus, $w_{i}$ has at least $\left|T^{\prime}\right|-d+1$ in-neighbours in $T^{\prime}$.

Our final lemma is obtained from iteratively applying Lemma 5.9 under the assumption that no subtournament is highly strongly-connected.

Lemma 5.10. Suppose $m \geq 2$ and $d \geq 1$ are integers, and suppose $T$ is a tournament such that $\delta^{+}(T) \geq d$ and no subtournament of $T$ is $m$-strongly-connected. Then there exists a set $S=\left\{w_{i} v_{i}: i \in[m]\right\}$ composed of pairwise vertex-disjoint directed edges and a set $F(S) \subseteq V(T)$ such that for every $i \in[\mathrm{m}]$

(1) $F(S) \cap(\bigcup S)=\varnothing$ and $|F(S)| \geq 2(d-4 m)$,

(2) $\left|N^{+}\left(v_{i}\right) \cap F(S)\right| \geq|F(S)|-m$, and

(3) $\left|N^{-}\left(w_{i}\right) \cap F(S)\right| \geq|F(S)|-d$.

Proof. We iteratively apply Lemma 5.9. Begin by removing vertices from $T$ such that the remaining tournament $T_{0}$ is vertex-minimal with $\delta^{+}\left(T_{0}\right) \geq d$. Then, since $T_{0}$ is not $m$-stronglyconnected by assumption, Lemma 5.9 implies that there is a positive integer $t_{1}<m$, a set of edges $S_{1}=\left\{w_{i}^{1} v_{i}^{1}: i \in\left[t_{1}\right]\right\}$, and a subtournament $F_{1} \subseteq T_{0}$ such that

- $V\left(F_{1}\right) \cap\left(\bigcup S_{1}\right)=\varnothing$,

- $\delta^{+}\left(F_{1}\right) \geq d-2 t_{1}$, and

- $\left|N^{+}\left(v_{i}^{1}\right) \cap F_{1}\right| \geq\left|F_{1}\right|-m$ and $\left|N^{-}\left(w_{i}^{1}\right) \cap F_{1}\right| \geq\left|F_{1}\right|-d$ for all $i \in\left[t_{1}\right]$.

We proceed by applying Lemma 5.9 inside $F_{1}$. Now assume we have already found subtournaments $F_{1} \supset \cdots \supset F_{k}$ with $\delta^{+}\left(F_{k}\right) \geq d-2 \sum_{i=1}^{k} t_{i}$, and disjoint sets of edges $S_{1}, \ldots, S_{k}$ of sizes $t_{1}, \ldots, t_{k}$, such that for each $j \in[k]$ we have $\left|N^{+}\left(v_{i}^{j}\right) \cap F_{k}\right| \geq\left|F_{k}\right|-m$ and $\left|N^{-}\left(w_{i}^{j}\right) \cap F_{k}\right| \geq\left|F_{k}\right|-d$ for all $i \in\left[t_{k}\right]$.

Assume that $\sum_{i=1}^{k} t_{i}<m$. We first find a vertex-minimal subtournament $T_{k}$ of $F_{k}$ such that $\delta^{+}\left(T_{k}\right) \geq d-2 \sum_{i=1}^{k} t_{i}$. Since by assumption $T_{k}$ is not $m$-strongly-connected, Lemma 5.9 implies that there is $t_{k+1}<m$, a non-empty set of edges $S_{k+1}=\left\{w_{i}^{k+1} v_{i}^{k+1}: i \in\left[t_{k+1}\right]\right\}$, and a subtournament $F_{k+1} \subseteq T_{k}$ such that

- $V\left(F_{k+1}\right) \cap\left(\bigcup S_{i}\right)=\varnothing$ for $i \in[k+1]$,

- $\delta^{+}\left(F_{k+1}\right) \geq \delta^{+}\left(T_{k}\right)-2 t_{k+1} \geq d-2 \sum_{i=1}^{k+1} t_{i}$

- $\left|N^{+}\left(v_{i}^{j}\right) \cap F_{k+1}\right| \geq\left|F_{k+1}\right|-m$ and $\left|N^{-}\left(w_{i}^{j}\right) \cap F_{k+1}\right| \geq\left|F_{k+1}\right|-d$ for $j \in[k+1]$ and $i \in\left[t_{j}\right]$.

We stop this process at a step $k_{0}$ when $\sum_{i=1}^{k_{0}} t_{i} \geq m$, and let

$$
S=\bigcup_{i=1}^{k_{0}} S_{i} \quad \text { and } \quad F(S)=F_{k_{0}} .
$$

Then clearly properties (2) and (3) of the claim are satisfied, and we have a set $S$ of at least $m$ edges which are all disjoint from $F(S)$. We just need to show that $|F(S)| \geq 2(d-4 m)$. Since $\sum_{i=1}^{k_{0}-1} t_{i}<m$ and $t_{k_{0}}<m$, we have that $\sum_{i=1}^{k_{0}} t_{i}<2 m$, and so $\delta^{+}\left(F_{k_{0}}\right) \geq d-4 m$. Thus,

$$
|F(S)|=\left|F_{k_{0}}\right| \geq 2 \delta^{+}\left(F_{k_{0}}\right) \geq 2(d-4 m)
$$

as claimed.

We are now in position to prove Theorem 1.3.

Proof of Theorem 1.3. As we already mentioned, the tightness can be seen by considering a random tournament on $\Omega(r \sqrt{\log r})$ vertices.

Now, let $C^{\prime}$ be the constant given by Lemma 5.8 and let $c$ be the constant given by Theorem 5.6. Put $C_{0}^{\prime}=\max \left\{C^{\prime}, 62 c\right\}$ and $C=1600 C_{0}^{\prime}$. Define $m=C^{\prime} r \sqrt{\log r}$ and $d=C r \sqrt{\log r}$, and suppose $T$ is a tournament with $\delta^{+}(T) \geq d$. Note that $C \geq 1600 C^{\prime}$. If $T$, or any subtournament of $T$, is $m$-strongly-connected, then we are done by Lemma 5.8; so we may assume 
otherwise. In this situation, we may apply Lemma 5.10. Indeed, let $S$ and $F(S)$ be given as in Lemma 5.10, and let $N_{i}=N^{-}\left(w_{i}\right) \cap F(S), M_{i}=N^{+}\left(v_{i}\right) \cap F(S)$, and $L_{i}=M_{i} \cap N_{i}$. We first observe that for each $i \in[\mathrm{m}]$

$$
\left|L_{i}\right| \geq|F(S)|-d-m \geq d / 2-m \geq d / 4,
$$

using the fact that $|F(S)| \geq 2(d-4 m)$. For every $i \in[m]$ choose a vertex $z_{i}$ from $L_{i}$ uniformly at random with replacement and let $L$ denote the resulting random set of vertices. Note that $\Delta_{i}:=\left\{w_{i}, v_{i}, z_{i}\right\}$ forms a directed triangle. Let $X$ denote the random variable recording the size of a largest subset of $L$ in which all vertices are pairwise distinct. Further, let $X^{\prime}$ count the number of pairs $\left\{z_{i}, z_{j}\right\}$ with $i \neq j$ and $z_{i}=z_{j}$. We have that $\mathbb{P}\left(z_{i}=z_{j}\right)=\left|L_{i} \cap L_{j}\right| /\left|L_{i}\right|\left|L_{j}\right| \leq$ $4 / d$ using (5.1). It follows that $\mathbb{E}\left[X^{\prime}\right] \leq\left(\begin{array}{c}m \\ 2\end{array}\right) \frac{4}{d} \leq 2 m^{2} / d$, and hence

$$
\mathbb{E}[X] \geq m-\mathbb{E}\left[X^{\prime}\right] \geq m-2 m^{2} / d=\left(C^{\prime}-2 C^{\prime 2} / C\right) r \sqrt{\log r} \geq\left(C^{\prime} / 2\right) r \sqrt{\log r}=m / 2,
$$

since $C \geq 1600 C^{\prime}$.

We say that a pair $\left\{\Delta_{i}, \Delta_{j}\right\}$ of directed triangles is good if there are edges between them in both directions; otherwise, we say this pair is bad. Observe that

$$
\begin{aligned}
\mathbb{P}\left(\left\{\Delta_{i}, \Delta_{j}\right\} \text { is bad }\right) & \leq \mathbb{P}\left(z_{j} \notin N^{+}\left(v_{i}\right) \cap F(S) \text { or } z_{i} \notin N^{+}\left(v_{j}\right) \cap F(S)\right) \\
& \leq \frac{m}{\left|L_{j}\right|}+\frac{m}{\left|L_{i}\right|} \leq \frac{8 m}{d}=\frac{8 C^{\prime}}{C} \leq \frac{1}{200},
\end{aligned}
$$

using (5.1) and since $C \geq 1600 C^{\prime}$. Hence, letting $Y$ denote the random variable counting the number of bad pairs of directed triangles, we have that

$$
\mathbb{E}[Y] \leq\left(\begin{array}{c}
m \\
2
\end{array}\right) \frac{1}{200} \leq m^{2} / 400
$$

Combining (5.2) and (5.3), and using the Cauchy-Schwarz inequality, we have

$$
\mathbb{E}\left[X^{2}-40 Y-\frac{m^{2}}{9}\right] \geq \mathbb{E}[X]^{2}-40 \mathbb{E}[Y]-\frac{m^{2}}{9} \geq \frac{m^{2}}{4}-\frac{m^{2}}{10}-\frac{m^{2}}{9}>0 .
$$

Accordingly, there is a choice of vertices from $\bigcup_{i=1}^{m} L_{i}$ such that $X^{2}-40 Y-m^{2} / 9>0$. It follows that $X^{2} \geq m^{2} / 9$, and so $X \geq m / 3$; i.e., there is a set $U$ of at least $m / 3$ vertices and they are all pairwise distinct. By possibly passing to a subset, let us assume $|U|=\lfloor m / 3\rfloor$. Moreover, we must have $40 Y \leq X^{2} \leq m^{2}$, and so the number of bad pairs of triangles is at most $m^{2} / 40$.

Our final aim is define a suitable auxiliary graph on our directed triangles and apply Theorem 5.6. Define a graph $G$ in the following way: put $V(G)=\left\{\Delta_{i}: z_{i} \in U\right\}$ and join $\Delta_{i}$ to $\Delta_{j}$ if and only if the pair $\left\{\Delta_{i}, \Delta_{j}\right\}$ is good. By our above analysis, we have $|V(G)|=\lfloor m / 3\rfloor$, and there are at most $m^{2} / 40$ non-edges in $G$. Thus, there are at least

$$
\left(\begin{array}{c}
\lfloor m / 3\rfloor \\
2
\end{array}\right)-m^{2} / 40 \geq m^{2} / 36-m^{2} / 40 \geq .0027 m^{2}
$$

edges in $G$, and so the average degree is at least $.0162 m \geq .0162(62 c r \sqrt{\log r})>c r \sqrt{\log r}$. Here we are using the fact that $m=C^{\prime} r \sqrt{\log r}$ and $C^{\prime} \geq 62 c$ by definition. Applying Theorem 5.6 to $G$, we have that $G$ contains a $K_{r}$ minor. It is easy to see that this $K_{r}$ minor corresponds to a strong $\vec{K}_{r}$ minor in the original tournament. This completes the proof of Theorem 1.3.

\section{STRONG MINORS IN DIGRAPHS}

We already know that large out-degree is, in general, not sufficient to guarantee large complete minors in digraphs. In this section, we give a positive result in digraphs for dichromatic number. More precisely, we prove Theorem 1.5, showing that there is a function $f(r)$ such that any digraph with dichromatic number at least $f(r)$ contains a strong $\vec{K}_{r}$ minor. First, we need a few definitions. A $\vec{K}_{r}$-template is a digraph $D$ such that $V(D)$ admits a partition into sets $X_{1}, \ldots, X_{r}$ such that for every ordered pair $(i, j)$ with $i \neq j \in[r]$, there is an edge from $X_{i}$ to 
$X_{j}$. We say that a digraph contains a $\vec{K}_{r}$-template if it contains a $\vec{K}_{r}$-template as a subdigraph. It is easy to find $\vec{K}_{r}$-templates in digraphs with high dichromatic number:

Lemma 6.1. Let $r \geq 1$ be an integer and suppose $D$ is a digraph with $\chi(D) \geq r$. Then $D$ contains a $\vec{K}_{r}$-template.

Proof. Let $A$ be a maximum sized transitive set in $D$ and let $D^{\prime}=D-A$. Then every vertex in $D^{\prime}$ has an out-neighbour and an in-neighbour in $A$ (otherwise, we could create a larger transitive set in $D$ ). Moreover, $\chi\left(D^{\prime}\right) \geq r-1$. The result follows by induction on $r$, by taking the union of a $\vec{K}_{r-1}$-template in $D^{\prime}$ together with the set $A$.

We are now ready to prove Theorem 1.5. The proof follows the ideas of Aboulker et al. [1] for embedding subdivisions of $\vec{K}_{r}$ in digraphs of high dichromatic number.

For a strongly-connected digraph $D$ we define an out-BFS-tree $T^{+}=T_{v}^{+}$in $D$ with root $v$ as a subdigraph of $D$ spanning $V(D)$, such that $T_{v}^{+}$is an oriented tree and for every $w \in V(D)$ we have $\operatorname{dist}_{T^{+}}(v, w)=\operatorname{dist}_{D}(v, w)$. Similarly, an in-BFS-tree $T^{-}=T_{v}^{-}$rooted at $v$ is a subdigraph of $D$ spanning $V(D)$ which is an oriented tree, such that for every $w \in V(D)$ we have $\operatorname{dist}_{T^{-}}(w, v)=\operatorname{dist}_{D}(w, v)$. For every integer $i$ and for a vertex $v \in V(D)$, let $L_{i}^{+, v}$ denote the $i$ th out-layer from $v$ in $D$, i.e. $L_{i}^{+, v}=\left\{w \in V(D): d_{D}(v, w)=i\right\}$. Similarly, $L_{i}^{-, v}$ denotes the $i$ th in-layer from $v$ in $D$, i.e. $L_{i}^{-, v}=\left\{w \in V(D): d_{D}(w, v)=i\right\}$.

We need the following simple fact:

Lemma 6.2. Let $D$ be a strongly connected digraph and let $T$ be an in-or out-BFS-tree in $D$. Let $X$ be any subset of vertices of $D$. Then there is a layer $L$ of $T$ such that $\chi(D[L \cap X]) \geq$ $\chi(D[X]) / 2$.

Proof. Without loss of generality, suppose that $T$ is an out-BFS-tree in $D$ with layers $L_{1}, \ldots, L_{m}$. Let $D_{1}$ and $D_{2}$ be the subdigraphs of $D$ induced by the odd and even layers of $T$, respectively. Since there is no arc from $L_{i}$ to $L_{j}$ for $j>i+1$, the strongly-connected components of $D_{1}$ and $D_{2}$ must be contained within the layers. It follows that $\chi\left(D_{1}[X]\right)=\max _{i \text { odd }} \chi\left(D\left[L_{i} \cap X\right]\right)$ and $\chi\left(D_{2}[X]\right)=\max _{i \text { even }} \chi\left(D\left[L_{i} \cap X\right]\right)$. Since $D$ is strongly-connected, we have $V(D)=V\left(D_{1}\right) \cup V\left(D_{2}\right)$ and and thus, $\chi(D[X]) \leq \chi\left(D_{1}[X]\right)+\chi\left(D_{2}[X]\right) \leq 2 \max _{i \in[m]} \chi\left(L_{i} \cap X\right)$.

Proof of Theorem 1.5. Let $D$ be a digraph such that $\chi(D) \geq r 4^{r}$. We shall show that $\operatorname{sm}(D) \geq r$. We may assume that $D$ is strongly-connected, otherwise, by Lemma 3.2, pass to a stronglyconnected component of high dichromatic number. For an integer $m$ with $0 \leq m \leq r$, an $m$ partial strong $\vec{K}_{r}$ minor in a digraph $D$ is a collection of pairwise disjoint subsets $X_{1}, \ldots, X_{r} \subseteq$ $V(D)$ such that

- for every pair $(i, j)$ with $i \neq j \in[r]$, there exists an edge from $X_{i}$ to $X_{j}$, and

- $D\left[V_{i}\right]$ is strongly-connected for at least $m$ sets $V_{i}$.

Note that a $\vec{K}_{r}$-template is a 0 -partial strong $\vec{K}_{r}$ minor. We define $f_{r}(m)$ to be the smallest $k$ such that any digraph with $\chi(D) \geq k$ contains an $m$-partial strong $\vec{K}_{r}$ minor. Note that by Lemma $6.1, f_{r}(0) \leq r$.

Claim 1. For any $m, 0 \leq m \leq r, f_{r}(m) \leq r 4^{m}$.

Claim 1 implies in particular that any digraph with dichromatic number at least $r 4^{r}$ has a strong $\vec{K}_{r}$ minor. We shall prove Claim 1 by induction on $m$. Let $\chi(D) \geq r 4^{m}, 0 \leq m \leq r$. If $m=0$, then the claim follows by Lemma 6.1. So assume that $1 \leq m \leq r$ and that the claim holds for smaller $m$. Let $v \in D$, let $T^{-}, T^{+}$be the in- and out-BFS-trees rooted at $v$, respectively.

Apply Lemma 6.2 to $D$ with $T=T^{-}$and $X=V(D)$ first to find a layer $L$ in $T=T^{-}$such that $\chi(D[L]) \geq \chi(D) / 2$. Then apply Lemma 6.2 to $D$ with $T=T^{+}$and $X=L$ to find a layer $L^{\prime}$ in $T^{+}$such that $\chi\left(D\left[L^{\prime} \cap L\right]\right) \geq \chi(D[L]) / 2$. Thus we have that $\chi\left(D\left[L^{\prime} \cap L\right]\right) \geq \chi(D) / 4 \geq r 4^{m-1}$. By induction, we have that $f_{r}(m-1) \leq r 4^{m-1}$, thus we can find an $(m-1)$-partial strong $\vec{K}_{r}$ minor 
$K$ contained in $L^{\prime} \cap L$ with branch sets $V_{1}, \ldots, V_{r}$. If all sets $V_{i}$ induce a strongly connected digraph, we have that $K$ is a strong $\vec{K}_{r}$-minor and $\operatorname{sm}(D) \geq r$, so we are done. Otherwise assume without loss of generality that $D\left[V_{1}\right]$ is not strongly-connected. Let $V_{1}=\left\{x_{1}, \ldots, x_{s}\right\}$. Now for each $\ell \in[s]$ let $P_{\ell}^{+}$be the directed $v-x_{\ell}$ path in $T^{+}$and $P_{\ell}^{-}$the directed $x_{\ell}-v$ path in $T^{-}$. Note that these paths "leave" the respective layers in $T^{+}$and $T^{-}$immediately. Then for $\ell \in[s],\left\{x_{\ell}\right\} \cup\left(P_{\ell}^{-}\right) \cup\left(P_{\ell+1}^{+}\right) \cup\left\{x_{\ell+1}\right\}$ (with addition modulo $s$ ) induces an $x_{\ell}-x_{\ell+1}$ walk, which contains an $x_{\ell}-x_{\ell+1}$ path. This path intersects $K$ exactly in the two points $\left\{x_{\ell}, x_{\ell+1}\right\}$, since $V(K)$ is contained in a layer $L$ of $T^{-}$and in a subset $L^{\prime}$ of a layer of $T^{+}$. Thus, letting $V_{1}^{\prime}=V_{1} \cup \bigcup_{\ell=1}^{s} P_{\ell}^{+} \cup P_{\ell}^{-}$, we see that $D\left[V_{k}^{\prime}\right]$ is strongly-connected and intersects $K$ only in $V_{1}$. Hence we obtain an $m$-partial strong $\vec{K}_{r}$ minor $K^{\prime}$ with branch sets $V_{1}^{\prime}, V_{2}, \ldots, V_{r}$. This proves the claim and the theorem.

\section{Concluding Remarks AND OPEn PROBlems}

We have investigated several relationships between large dichromatic number and the presence of strong complete minors in digraphs. Many problems remain. Regarding Theorem 1.2, the most obvious question is whether or not the bound on dichromatic number can be decreased.

Question 7.1. Is it true that any tournament $T$ with $\chi(T) \geq(1+o(1)) r$ satisfies $\operatorname{sm}(T) \geq r$ ?

For general digraphs, our bounds are far apart. To take a more general view, we may address the following question: Which digraph parameters force the existence of large strong complete minors? To formalize this, for a digraph $H$ and a digraph parameter $\phi$, we let $\operatorname{sm}_{\phi}(H)$ denote the smallest integer such that any digraph $D$ with $\phi(D) \geq \operatorname{sm}_{\phi}(H)$ contains a strong $H$ minor; if such an integer does not exist, then we define $\operatorname{sm}_{\phi}(H)=\infty$. This is analogous to the work of Aboulker et al. [1], where they introduced the parameter $\operatorname{mader}_{\phi}(H)$ as the smallest integer such that any digraph $D$ with $\phi(D) \geq \operatorname{mader}_{\phi}(H)$ contains a subdivision of $H$. Theorem 1.4 can be expressed as saying that for any $r, \operatorname{sm}_{\delta^{+}}\left(\vec{K}_{r}\right)=\infty$, and our Theorem 1.5 can be expressed succintly as

$$
r+1 \leq \operatorname{sm}_{\chi}\left(\vec{K}_{r}\right) \leq r 4^{r} .
$$

It would be very interesting to obtain better bounds on this function.

In Lemma 5.8, we showed that strong-connectivity is sufficient to guarantee large complete minors in tournaments. Is the same true in general digraphs? Letting $\kappa(D)$ denote the strongconnectivity of a digraph $D$, we pose the following problem:

Problem 7.2. Determine whether or not $\operatorname{sm}_{\kappa}\left(\vec{K}_{r}\right)<\infty$.

For a given natural digraph parameter $\phi$, it would be interesting to determine $\operatorname{sm}_{\phi}(H)$, for some other digraphs $H$, for example oriented trees, cycles, or transitive tournaments.

\section{REFERENCES}

[1] P. Aboulker, N. Cohen, F. Havet, W. Lochet, P. F. Moura, and S. Thomassé, Subdivisions in digraphs of large out-degree or large dichromatic number, Electronic Journal of Combinatorics 26 (2019), no. 3.

[2] E. Berger, K. Choromanski, M. Chudnovsky, J. Fox, M. Loebl, A. Scott, P. Seymour, and S. Thomassé, Tournaments and colouring, Journal of Combinatorial Theory, Series B 103 (2013), no. 1, 1-20.

[3] B. Bollobás, P. A Catlin, and P. Erdős, Hadwiger's conjecture is true for almost every graph., Eur. J. Comb. 1 (1980), no. 3, 195-199.

[4] G. A Dirac, A property of 4-chromatic graphs and some remarks on critical graphs, Journal of the London Mathematical Society 1 (1952), no. 1, 85-92.

[5] A. Girão, K. Popielarz, and R. Snyder, Subdivisions of digraphs in tournaments, Journal of Combinatorial Theory, Series B 146 (2021), 266-285.

[6] L. Gishboliner, R. Steiner, and T. Szabó, Dichromatic number and forced subdivisions, arXiv preprint arXiv:2008.09888 (2020).

[7] _ Oriented cycles in digraphs of large outdegree, arXiv preprint arXiv:2008.13224 (2020).

[8] H. Hadwiger, Über eine Klassifikation der Streckenkomplexe, Vierteljschr. Naturforsch. Ges. Zürich 88 (1943), no. $2,133-142$

[9] A. Harutyunyan, T.-N. Le, A. Newman, and S. Thomassé, Coloring Dense Digraphs, Combinatorica 39 (2019), 1021-1053. 
[10] A. Harutyunyan, T.-N. Le, S. Thomassé, and H. Wu, Coloring tournaments: From local to global, Journal of Combinatorial Theory, Series B 138 (2019), 166-171.

[11] C. Jagger, An extremal function for digraph subcontraction, Journal of Graph Theory 21 (1996), no. 3, 343-350.

[12] _ Extremal digraph results for topological complete subgraphs, European Journal of Combinatorics 19 (1998), no. 6, 687-694.

[13] T. Johnson, N. Robertson, P. D. Seymour, and R. Thomas, Directed Tree-Width, Journal of Combinatorial Theory, Series B 82 (2001), no. 1, 138-154.

[14] I. Kim and P. Seymour, Tournament minors, Journal of Combinatorial Theory, Series B 112 (2015), 138-153.

[15] A. V. Kostochka, Lower bound of the Hadwiger number of graphs by their average degree, Combinatorica 4 (1984), no. 4, 307-316.

[16] V. Neumann-Lara, The Dichromatic Number of a Digraph, Journal of Combinatorial Theory, Series B 33 (1982), 265-270.

[17] S. Norin and Z.-X. Song, Breaking the degeneracy barrier for coloring graphs with no $K_{t}$ minor, arXiv preprint arXiv:1910.09378 (2019).

[18] A. Pokrovskiy, Highly linked tournaments, Journal of Combinatorial Theory, Series B 115 (2015), 339-347.

[19] L. Postle, Halfway to Hadwiger's Conjecture, arXiv preprint arXiv:1911.01491 (2019).

[20] _ Further progress towards Hadwiger's conjecture, arXiv preprint arXiv:2006.11798 (2020).

[21] N. Robertson, P. Seymour, and R. Thomas, Hadwiger's conjecture forK 6-free graphs, Combinatorica 13 (1993), no. 3, 279-361.

[22] A. Thomason, An extremal function for contractions of graphs, Mathematical Proceedings of the Cambridge Philosophical Society, 1984, pp. 261-265.

[23] C. Thomassen, Even cycles in directed graphs, European Journal of Combinatorics 6 (1985), no. 1, 85-89.

[24] K. Wagner, Über eine Eigenschaft der ebenen Komplexe, Mathematische Annalen 114 (1937), no. 1, 570-590. 